\title{
Towards public health surveillance of intensive care services in NSW, Australia
}

\section{Sophie Nortona,b,e, Damien V Corderya ${ }^{a}$, Brett J Abbenbroek ${ }^{c}$, Angela C Ryan $^{c}$ and David J Muscatello ${ }^{a, d}$}

a Centre for Epidemiology and Evidence, NSW Ministry of Health, Sydney, Australia

b Western Sydney Local Health District Public Health Unit, NSW, Australia

${ }^{c}$ eHealth NSW, Sydney, Australia

d School of Public Health and Community Medicine, University of New South Wales, Sydney, Australia

e Corresponding author: sophie.norton@health.nsw.gov.au

\section{Article history}

Publication date: July 2016

Citation: Norton S, Cordery DV,

Abbenbroek BJ, Ryan AC, Muscatello DJ.

Towards public health surveillance

of intensive care services in NSW,

Australia. Public Health Res Pract.

2016;26(3):e2631633. doi: http://dx.doi.

org/10.17061/phrp2631633

\section{Key points}

- A mainstream, continuous, rapid intensive care unit (ICU) surveillance facility that will readily adapt to emergency situations would be a valuable resource for protecting population health

- A new intensive care clinical information system being developed for New South Wales ICUs offers this opportunity

- A syndromic intensive care surveillance system approach would best achieve stakeholders' ICU surveillance objectives

\section{Abstract}

Objectives: Outbreaks of known and novel pathogens causing very severe illness increase the risk to public health in a globalised community and alarm the public. Intensive care units (ICUs) may be an underused setting for public health surveillance. This study investigates the electronic Record for Intensive Care (eRIC), an electronic clinical information and management system being developed for New South Wales ICUs, and its surveillance opportunity offerings.

Methods: The surveillance benefits being introduced by the eRIC were evaluated through consultation with stakeholders and the eRIC program team. The consultation process involved providing stakeholders with background information about the eRIC system. Based on the consultation, a draft data and information model for surveillance was developed. The model was evaluated using guidelines from the US Centers for Disease Control and Prevention.

Results: Population health stakeholders confirmed that the eRIC offers an appealing surveillance data source for pathogens and other hazards causing severe illness. Suggested application of the surveillance included, for known hazards, seasonal and pandemic influenza, enterovirus 71, Murray Valley encephalitis virus, enterohaemorrhagic Escherichia coli 0104:H4 and parechovirus. The proposed surveillance model uses syndromic rather than specific-cause surveillance. It may offer greater timeliness and sensitivity than relying on reporting of diagnoses of specific pathogens. Five syndromes derived from clinical pathways in the eRIC are proposed: severe acute respiratory disease, severe acute neurological disease, sepsis or septicaemia, jaundice or hepatitis, and acute renal failure.

Conclusion: New intensive care clinical information systems offer a largely untapped resource for continuous, mainstream, rapid ICU surveillance of severe illness. A continuous, mainstream, rapid ICU surveillance facility that will readily adapt to emergency situations would be a valuable resource for protecting population health. This study establishes a firm basis on which ICU surveillance can be developed. 


\section{Introduction}

Over the past two decades, public concern has grown about global public health risks and health emergencies related to communicable diseases, natural disasters, and intentional or accidental release of biological and chemical material. Of the numerous causes of acute communicable disease, a priority is placed on pathogens with pandemic potential and those causing sudden severe illness. Novel respiratory viruses such as H1N1 influenza virus, Middle East respiratory syndrome coronavirus (MERS-CoV) and avian influenza A (H7N9) virus $^{1-3}$ have caused high morbidity and mortality, and economic strain, worldwide. Public health surveillance, "the ongoing systematic collection, analysis, and interpretation of health data that are essential to the planning, implementation, and evaluation of public health practice" 4 , is integral to identifying and controlling these risks. The International Health Regulations (IHR-2005) emphasise "the timely dissemination of public health information for assessment and public health response". ${ }^{5}$

Intensive care units (ICUs) may be an underused setting for public health surveillance of pathogens causing severe illness. The $2009 \mathrm{H} 1 \mathrm{~N} 1$ influenza pandemic highlighted the impact that a novel respiratory virus can have on naive populations, when approximately $22 \%$ of hospital admissions for influenza in Australia led to an ICU admission. ${ }^{3}$ Subsequent assessment of surveillance systems employed in the state of New South Wales (NSW), Australia, during this time pointed to a need for more timely ICU surveillance, with minimal impact on clinicians facing a surge in workload. ${ }^{3,6}$ NSW developed two internet-accessible form-based databases for ICU surveillance during the 2009 H1N1 influenza pandemic. ${ }^{7}$ In 2007, NSW also implemented the Critical Care Resource management System (CCRS) for real-time management of demand and patient interhospital transfers. The CCRS provided limited patient surveillance capability.

NSW is implementing a state-wide standardised electronic health care record and patient management system in ICUs called the electronic Record for Intensive Care (eRIC). In coming years, it is intended that the eRIC will be rolled out to the majority of NSW adult, paediatric and neonatal ICUs. This development offers a unique opportunity to establish continuous, mainstream, rapid ICU surveillance in NSW.

\section{Overview of the NSW electronic Record for Intensive Care system}

\section{System architecture}

The eRIC information system will be a single point of collated patient data, receiving direct input from bedside medical devices (e.g. haemodynamic monitors, ventilators, infusion devices) and clinical systems (e.g. laboratory, radiology, electronic medical record), and will process this information to assist with clinical decision making. ${ }^{8}$

The eRIC will be integrated with software used for the collection of information required by the Australian and New Zealand Intensive Care Society (ANZICS) Adult Patient Database (APD), and the Australian and New Zealand Paediatric Intensive Care (ANZPIC) Registry. ${ }^{9}$ This information, organised and classified for research purposes, may provide rich surveillance information.

Clinical information will be recorded primarily in a structured format (selecting from options) and as free text. Systematised Nomenclature of Medicine - Clinical Terms (SNOMED CT), a medical encoding ontology ${ }^{10}$, will be used for recording structured information that information systems can interpret and process. It is expected that a SNOMED CT-coded admission diagnosis will be available in the eRIC early in the ICU admission. The eRIC will implement the Acute Physiology and Chronic Health Evaluation (APACHE) II and III diagnosis classification system, a comprehensive list of postoperative and nonoperative conditions ${ }^{11}$, which is used by ANZICS.

\section{Patient demographics}

The eRIC will interface with the wider hospital patient administration system, from which it will extract demographic information such as age, gender and residential locality of the patient.

\section{Clinical parameters}

Bedside devices will automatically record vital signs and other physiological parameters on patients' individual clinical observation flowcharts integrated in the eRIC. Clinicians will verify information and add other therapeutic details. Specific cells on the observation chart will be populated as therapies commence.

Structured clinical protocols will be built into the eRIC and used interactively by clinicians, and the resulting information will be integrated into the patient's eRIC record. Operational reports such as lists of patients on specific therapies will be compiled.

\section{Risk factors}

APACHE II and III diagnosis and subdiagnosis codes, and APACHE chronic health evaluation scores based on pre-existing conditions ${ }^{11}$, available as SNOMED CT coding, will provide structured risk-factor information.

This study evaluated the potential public health surveillance benefits of the eRIC. Forward planning can be valuable to ensure that fields are defined and built before implementation. Based on consultation, a draft data and information model of the system was developed, and evaluated according to standard guidelines. ${ }^{12}$

\section{Methods}

From May to October 2013, the eRIC was evaluated for its potential use in public health surveillance. 


\section{Consultation with potential users of a public health surveillance system}

A range of meetings were held with the following NSW population health stakeholders: the NSW Infectious Diseases Network; the Public Health Informatics and Epidemiology Network; and the Directors of Health Protection, Communicable Diseases and Environmental Health in NSW. Background eRIC information was provided before consultation. Stakeholders were asked to give feedback - which they provided verbally at meetings and in written format - on what value this information could provide for public health surveillance and reporting purposes. A second round of consultation was undertaken with stakeholders from Health Protection NSW in August 2014, to assess the usefulness (from a surveillance perspective) of adding unique indicators to the standard ICU clinical data collection.

\section{Drafting a design for the system}

A set of objectives for a public health surveillance system was produced, informed by the consultation process. A model for the surveillance system was proposed, based on objectives and available eRIC information.

\section{Evaluation of the proposed surveillance model}

The proposed eRIC-based model was evaluated for its potential capacity as a data source for public health surveillance. It was assessed against the attributes of a good public health surveillance system, as described by the US Department of Health and Human Services, Centers for Disease Control and Prevention. ${ }^{12}$

\section{Results}

\section{Consultation with potential users}

In the first consultation round, approximately 25 people were involved in face-to-face discussions through the two network meetings. Multiple public health unit (PHU) representatives from all 15 NSW Local Health Districts (LHDs) were reached via the NSW Infectious Diseases Network teleconference. A further five key health protection stakeholders - the Director of Health Protection NSW, two medical epidemiologists, a manager of surveillance and a senior surveillance officer - were involved in a focused discussion with study investigators in the second round of consultation. The interviewees discussed current and emerging pathogens and conditions that may be of surveillance value, and treatment monitoring that indicates disease severity.

The participants indicated that their primary interests were to detect rare or new pathogens that have pandemic potential or cause severe disease (particularly severe respiratory disease), and the need to be prepared and to easily access ICU information during a pandemic.
Other pathogens of interest for surveillance were seasonal influenza ${ }^{13}$; enterohaemorrhagic Escherichia coli 0104: $\mathrm{H} 4^{14}$ causing haemolytic uraemic syndrome; and pathogens that cause neurological syndromes, such as enterovirus $71^{15}$, Murray Valley encephalitis virus ${ }^{16}$ and parechovirus ${ }^{17}$, as they can cause outbreaks of severe illness. There was interest in enhancing surveillance efforts for poliomyelitis ${ }^{18}$, and in monitoring influenza patterns and burden by reporting on positive influenza results and antiviral pharmacological information linked to ICU patients. Although it was recognised that there were processes for notification of diseases reportable under the Public Health Act 2010 (NSW) ${ }^{19}$, positive notifiable laboratory results linked to ICU patients would be valuable in monitoring for the emergence of more virulent strains and rarer syndromes, for which recognition and normal passive notification may be delayed. ${ }^{20}$

Stakeholders commonly stated that adding userentered surveillance-specific fields to standard ICU clinical data collection would result in poor entry compliance by staff, because the data would not be immediately clinically relevant. Reporting and feedback of surveillance data to ICUs and LHDs was identified as an important attribute of surveillance.

\section{Draft design of the system}

Based on the consultation, the objectives of the surveillance system will be to:

- Provide situational awareness during an outbreak or pandemic of a pathogen or other health risk that causes severe illness

- Provide early warning of increases in disease activity caused by novel or rarer pathogens that cause severe illness

- Detect probable cases, and associated demographics and comorbidities during an outbreak or pandemic of a known pathogen that causes severe illness

- Provide data during the influenza season that are indicative of disease severity and burden.

Syndromic surveillance, which monitors syndromes rather than specific diagnosis and signals clustering in time or geography ${ }^{21}$, is the suggested approach to achieve these objectives.

The proposed data and information model of the eRICbased surveillance system is outlined in Table 1. The eRIC will be used interactively in real time by clinicians and, thus, it should be feasible for the surveillance system to provide reports in near real time. The NSW Ministry of Health currently performs real-time data capture and near real-time analysis of surveillance information using hospital emergency department (ED) data. ${ }^{22}$ Figure 1 depicts a draft information flow for the system.

\section{Primary admission diagnosis-based syndromes}

As demonstrated in ED surveillance in NSW22, syndromes can be formed based on primary diagnoses from clinical information systems. Five syndrome groupings are 
Table 1. Proposed data and information model of the electronic Record for Intensive Care surveillance system

\begin{tabular}{lll}
\hline $\begin{array}{l}\text { Primary diagnosis } \\
\text { syndrome }\end{array}$ & $\begin{array}{l}\text { Significant clinical } \\
\text { pathway/intervention }\end{array}$ & Data source (trigger) \\
\hline $\begin{array}{lll}\text { Severe acute respiratory } \\
\text { disease (pneumonia, }\end{array}$ & ARDS, CRRT, sepsis, & $\begin{array}{l}\text { Primary syndrome: admission } \\
\text { diagnosis and updates from }\end{array}$ \\
$\begin{array}{l}\text { pneumonitis, asthma, } \\
\text { influenza, bronchiolitis) }\end{array}$ & & $\begin{array}{l}\text { ward-round information } \\
\text { Intervention: admission diagnosis } \\
\text { and assessment, or flowchart cell }\end{array}$
\end{tabular}

Severe acute neurological

disease (meningitis, encephalitis, acute flaccid

paralysis, Guillain-Barré

syndrome, acute brainstem infection)

Sepsis or septicaemia

Jaundice or hepatitis (fulminant hepatitis, hepatic failure)

Acute renal failure

\section{Sepsis, ECMO, ARF As above} (with CRRT)

ARF (with CRRT) As above

Coagulopathy, encephalopathy

CRRT, microangiopathic anaemia studies
Public health indication

Early warning of novel or rare respiratory viruses

Severity and burden of disease information during an outbreak (coronaviruses, influenza, respiratory syncytial virus)

Early warning of, and disease-burden information during, neurological-related outbreaks (enterovirus 71, Murray Valley encephalitis virus, parechovirus) Identification of acute flaccid paralysis and Guillain-Barré syndrome cases, indicating possible poliomyelitis

Burden of disease information related to infections caused by pathogens from any primary site (parechovirus)

Early identification of cases caused by infections of public health significance (hepatitis A, B, C and E) ${ }^{23}$ and those with environmental causes (e.g. mushroom poisoning) Intervention: Admission diagnosis and ward-round updates. Positive indicators on test results of full blood count and coagulation

CRRT - as above Blood film results
Burden of disease information related to infections caused by pathogens from any primary site, especially in an outbreak of shiga toxin-producing Escherichia coli, causing haemolytic uraemic syndrome

ARDS = acute respiratory distress syndrome; $\mathrm{ARF}=$ acute renal failure; $\mathrm{CRRT}=$ continuous renal replacement therapy; $\mathrm{ECMO}=$ extracorporeal membrane oxygenation

proposed: 'severe acute respiratory disease', 'severe acute neurological disease', 'sepsis', 'acute renal failure' and 'hepatitis'. Each will include groups of SNOMED CT diagnosis codes, which represent ailments of the same affected organ, but with myriad causes.

\section{Clinical pathway, intervention or risk factor-based syndromes}

Syndromes can be further defined by capturing clinical pathways (a predictable clinical course that the patient is taking), clinical interventions and risk factors (see Table 1). Examples include acute respiratory distress syndrome (ARDS), acute renal failure, continuous renal replacement therapy (CRRT) and extracorporeal membrane oxygenation (ECMO).

\section{Data items needed}

Diagnosis syndromes would be captured directly from the diagnosis coding collected at ICU admission. A future eRIC development could capture diagnosis updates from standardised ward-round templates. Significant clinical syndromes and commencement of significant clinical treatments could be captured from a single data point within individual observation charts or within protocols such as ARDSNet (a protocol for managing ventilation in patients with ARDS). Reports that are compiled by the eRIC would offer direct counts of patients experiencing interventions of interest. Risk-factor information should be captured from the patient's admission summary component of the eRIC record, and be available by enquiry when clustering requires further investigation.

\section{Evaluation of the proposed surveillance system \\ Usefulness}

A surveillance system that is established and automatic will give insight into the background incidence of monitored syndromes in specific hospital ICU populations. This will assist characterisation of an outbreak or pandemic by person, time and place at the beginning of the event when more targeted surveillance 
Figure 1. Draft information flow of the electronic Record for Intensive Care-based surveillance system<smiles>c1ccccc1</smiles>

Patient

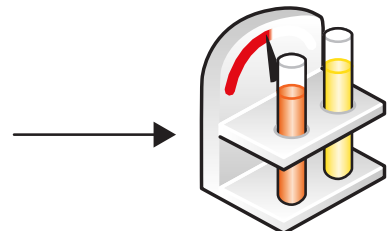

Medical devices
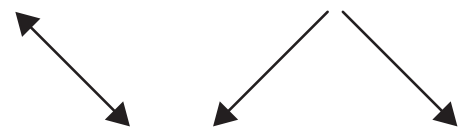

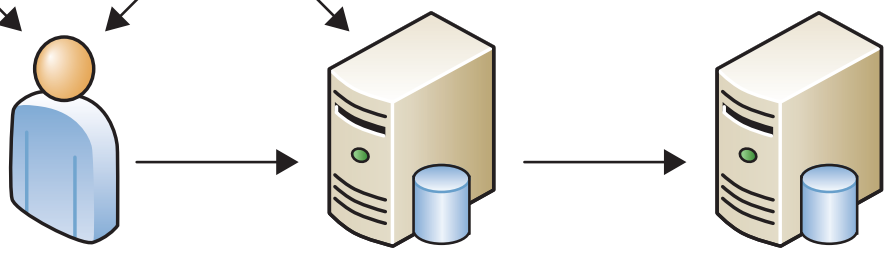

Health professional electronic Record for Intensive Care (eRIC)
Reporting database

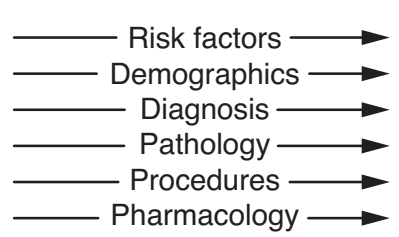

Pharmacology

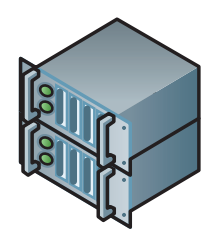

Data warehouse

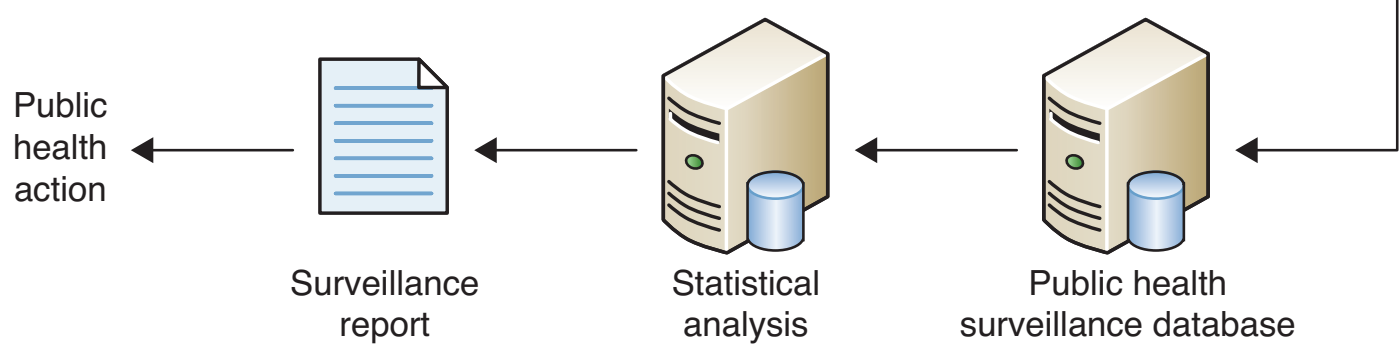

activities have not yet been established, as well as throughout the event.

\section{Simplicity}

Automated capture of routinely collected electronic data from the eRIC minimises personnel involvement. Keeping the data and its collection points minimal, uncomplicated and easily interpreted will allow the software and surveillance system to easily interpret the information.

The eRIC will be integrated with multiple hospital information systems, all of which are subject to failures. Therefore, the following will also be needed:

- A high degree of technical skill and complex infrastructure, to create the mechanisms for capturing data from the eRIC and feeding them to the public health surveillance system

- Protocols that can identify site-specific and wholesystem data-feed inconsistencies, and systems for managing these problems

- On-call support for a surveillance system that operates 7 days a week.

Uncertainty attached to interpreting syndromic surveillance data signals ${ }^{24}$ will require staff training to avoid unnecessary reporting and follow-up.

\section{Flexibility}

Reporting diagnoses by syndrome will allow detection of unusual illness patterns from a broad range of causes and clinical presentations. Increases in new illnesses can be captured without making changes to system configuration, and using configurable reference tables to define syndromes would allow changes and additions to syndromes without system reprogramming.

\section{Data quality}

Data will be more complete by using data fields from source information systems that are consistently and completely used by hospital staff, because they facilitate workflow and patient management. Admitting diagnoses should be consistently reported. Data validity would be improved by grouping related diagnoses by syndromes rather than a specific diagnosis, which could be influenced by the admitting physician's preference for diagnosis description.

Data-feed mechanisms can be designed to ensure that any data not saved during temporary data outages are autofilled on resumption of feeds or source systems. This can be done through synchronous data communication protocols or redundancy in the data periods included in batch extracts. Data governance and a data dictionary are important elements to ensure that data quality is maintained. ${ }^{24}$ 


\section{Acceptability}

Automation involves personnel only when a signal triggered by the surveillance system requires follow-up. Two-way communication is recognised as an important function of surveillance systems. ${ }^{25}$ Clinicians should understand that, if they provide their time, the outcomes and benefits can link back to improvements for their patients and the community. ${ }^{5}$ An efficient and effective feedback mechanism through LHD PHUs will be essential to establishing a strong relationship.

\section{Sensitivity}

Sensitivity can be considered in terms of the proportion of cases of a specific disease that are detectable by the surveillance system or the ability of surveillance to detect outbreaks. ${ }^{12}$ Broad-diagnosis syndromes are more inclusive of all cases relating to a single pathogen, because they account for variation in clinical manifestations and subjective differences in clinician diagnosis. This is important when the causative pathogen may not be known or anticipated, particularly for emerging infections. The improved outbreak detection will be restricted to outbreaks that can cause severe illness and ICU admission. This information would complement other established NSW ED and laboratory surveillance systems.

Using syndromic surveillance means that, as the magnitude of a signal increases, sensitivity increases and time to detection decreases relative to the baseline incidence ${ }^{26}$, meaning that smaller outbreaks could be missed. Initial disease increases in small regional populations could be missed when residents are transferred to out-of-area ICUs and numbers are absorbed into those counts. Increased sensitivity increases occurrence of false alarms. ${ }^{27}$ Surveillance team experience around usual and anticipated geographic, temporal and demographic patterns of disease will enhance interpretation of the surveillance information.

\section{Specificity}

Broad-diagnosis groupings will have low specificity for individual pathogens ${ }^{26}$; this is a trade-off for a high sensitivity that allows identification of pathogens that may not otherwise be recognised because of negative pathology test results, or inappropriate or no testing. It is important to use high-quality clinical information for clinical activities, which should ensure that the information recorded in the eRIC will lead to consistent identification of syndromes. This should reliably indicate where further follow-up is warranted, and clinicians can be prompted to undertake further investigations, such as microbiological testing, to search for novel pathogens. Including negative and positive pathology results in surveillance information should facilitate interpretation of surveillance signals. ICU clinical pathways should assist with identification of potential cases of an emerging pathogen. ARDS, often requiring $\mathrm{ECMO}$, has been a feature of many recently emerging novel pathogens. ${ }^{3,28}$

\section{Representativeness}

The public health intensive care surveillance system proposes to capture and inform public health about patterns of severe illness occurring in NSW. The eRIC will be implemented state-wide, with comprehensive rural and urban coverage, and will represent all age groups within NSW by including adult, paediatric and neonatal intensive care beds.

\section{Timeliness}

Timeliness should be high because of the anticipated near real-time data capture and analysis envisaged for the surveillance system.

\section{Stability}

Stability of the surveillance system would be dependent on the success of the eRIC and how it operates in the clinical setting - that is, how sustainable the information system is in practice, and its importance from a health system data warehousing and performance monitoring perspective. The eRIC will be hosted centrally and deployed virtually across NSW, with full disaster recovery and redundancy to minimise unscheduled downtime, and high-system availability planned for $99.9 \%$ of the time.

The need for design and implementation of the surveillance system to meet prespecified stability criteria before acceptance as a production facility will influence the stability of the systems for capture, analysis and reporting of surveillance data. Development of relationships with eRIC maintenance personnel, technical experts in troubleshooting data-capture problems and the eRIC 'business as usual' team will contribute stability.

\section{Discussion}

Stakeholders have identified that ICU surveillance data are an essential element of preparedness for severe disease outbreaks and pandemics. The data contribute important information about disease burden, illness severity and epidemiological characteristics. Improvement in ICU surveillance capacity in NSW is required..$^{3,6,7}$ As an integral tool of care in NSW ICUs, the eRIC will offer a large amount of diverse information about ICU patients that can be harnessed for public health surveillance.

The literature describes electronic reporting systems established primarily for retrospective audit, performance analysis and research purposes, such as the ANZICS Centre for Outcome and Resource Evaluation (CORE) ${ }^{9}$ registries. These systems are also used to assist with patient management for surveillance of nosocomial infections ${ }^{29}$, rather than being set up for routine public health surveillance. Although the ICU databases used in NSW during the $2009 \mathrm{H}_{1} \mathrm{~N} 1$ influenza pandemic ${ }^{7}$ are purpose built for public health surveillance, they are influenza specific and not timely because of the lack of automation. ANZICS CORE surveillance shares public 
health surveillance system objectives, such as support of "influenza pandemic planning, biosecurity and terrorism planning", but it requires only quarterly reporting by member ICUs across state, federal and international jurisdictions. The near real-time NSW public health surveillance system will offer a more streamlined capture of NSW-specific information, which will be reported in a manner that will be meaningful for early interpretation.

Assessment against standard guidelines supports the use of a syndromic surveillance approach to best achieve public health objectives. Syndromic surveillance has been increasingly used successfully in emergency management and preparedness over the past decade in non-ICU situations in Australia and internationally. ${ }^{22}$

The described surveillance approach could indicate concern through the following scenarios:

- A single patient diagnosed with an acute respiratory event, commenced on therapies such as ARDSNet or ECMO, with an untypeable influenza A result, indicating infection with a novel strain

- A daily ECMO report that reveals that the number of patients on ECMO is above expected numbers

- A patient with acute renal failure requiring CRRT with microangiopathic anaemia.

Continuous ICU surveillance may benefit other surveillance efforts. Sepsis is frequently seen in ICUs, with a large proportion of sepsis cases caused by respiratory infections acquired in a nonhospital setting. ${ }^{30}$ This surveillance system could contribute to understanding infection patterns already occurring in the NSW population.

A considerable period of time will be required to establish baseline trends in ICU-based surveillance syndromes, creating a challenge in identifying and interpreting unusual trends that could signify an outbreak. This should be mitigated through cautious interpretation and experience over time. Case definitions for syndrome groups will need to be re-evaluated at regular intervals to ensure their ongoing relevance and value. Once the system is implemented, it will be important to repeatedly evaluate it.

\section{Conclusion}

A mainstream, continuous, rapid ICU-based public health surveillance facility that will readily adapt to emergency situations will provide a valuable resource for protecting population health. This study identifies the key requirements and risks that need to be considered when establishing the system, and provides a firm basis on which to proceed when the eRIC is realised.

\section{Acknowledgements}

This work was completed while SN was a trainee on the NSW Public Health Training Program, funded by the NSW Ministry of Health. The authors also acknowledge the Centre for Epidemiology and Evidence, NSW Ministry of Health; the eRIC; and the Agency for Clinical Innovation Working Group.

\section{Competing interests}

None declared

\section{Author contributions}

SN was responsible for the design, drafting, data analysis and editing of the manuscript. DC was responsible for the design, data analysis and editing of the manuscript. BA and AR were responsible for providing advice on content, and reviewing and editing the manuscript. DM was responsible for supervising the project, and reviewing and editing the manuscript.

\section{References}

1. World Health Organization. Middle East respiratory syndrome coronavirus. Riyadh: WHO, Kingdom of Saudi Arabia; 2013 [cited 2013 Sep 19]. Available from: www. who.int/csr/disease/coronavirus_infections/en/

2. World Health Organization. Global alert and response. Human infection with avian influenza A(H7N9) virus. Geneva: WHO; 2013 [cited 2013 Sep 13]. Available from: www.who.int/csr/don/2013_08_11/en/index.html\#

3. Kelly PM, Kotsimbos T, Reynolds A, Wood-Baker R, Hancox B, Brown SG, et al. FluCAN 2009: initial results from sentinel surveillance for adult influenza and pneumonia in eight Australian hospitals. Med J Aust. 2011;194(4):169-74.

4. Remington PL, Nelson DE. Communicating public health surveillance information for action. In: Lee LM, Teutsch SM, Thacker SB, St Louis ME, editors. Principles and practice of public health surveillance, 3rd ed. New York: Oxford University Press; 2010. p. 146-65.

5. World Health Organization. International health regulations. Second edition. Geneva: WHO; 2005 [cited 2014 May 1]. Available from: www.who.int/ ihr/9789241596664/en/

6. Churches T, Conaty SJ, Gilmour RE, Muscatello DJ. Reflections on public health surveillance of pandemic (H1N1) 2009 influenza in NSW. N S W Public Health Bull. 2010; 21(1-2):19-25.

7. New South Wales public health network. Progression and impact of the first winter wave of the 2009 pandemic H1N1 influenza in New South Wales, Australia. Euro Surveill. 2009;14(42):pii=19365.

8. eHealth, NSW Government. Electronic Record for Intensive Care (eRIC). Sydney: eHealth, NSW Government; [cited 2016 May 23]. Available from: www. ehealth.nsw.gov.au/programs/clinical/eric

9. Australian and New Zealand Intensive Care Society. Centre for Outcome and Resource Evaluation (CORE). Melbourne: ANZICS; 2015 [cited 2015 Oct 25] [about 2 screens]. Available from: www.anzics.com.au/Pages/ CORE/About-CORE.aspx 
10. Shahpori R, Doig C. Systematized Nomenclature of Medicine-Clinical Terms direction and its implications on critical care. J Crit Care. 2010;25(2):364.e1-9.

11. Australian and New Zealand Intensive Care Society Centre for Outcome and Resource Evaluation. APD data dictionary for software programmers. ANZICS CORE adult patient database, version 4. Melbourne: ANZICS; 2013 [cited 2015 Oct 25]. Available from: www.anzics. com.au/Downloads/ANZICS\%20APD\%20Dictionary\%20 V4_Programmer.pdf

12. German RR, Lee LM, Horan JM, Milstein RL, Pertowski CA, Waller NM, et al. Updated guidelines for evaluating public health surveillance systems. Recommendations from the Guidelines Working Group. MMWR Recomm Rep. 2001;50(RR-13):1-35.

13. Schaffer A, Muscatello D, Cretikos M, Gilmour R, Tobin S, Ward J. The impact of influenza $A(H 1 N 1)$ pdm09 compared with seasonal influenza on intensive care admissions in New South Wales, Australia, 2007 to 2010: a time series analysis. BMC Public Health. 2012;12(869):1-14.

14. Frank C, Werber D, Cramer JP, Askar M, Faber M, Heiden M, et al. Epidemic profile of Shiga-toxinproducing Escherichia coli 0104:H4 outbreak in Germany. N Eng J Med. 2011;3659190:1771-80.

15. NSW Health Communicable Diseases. Enhanced surveillance for enterovirus-associated neurological disease in children. Report 8. Sydney: NSW Health; 2013 [cited 2013 Sep 17]. Available from: www.health.nsw. gov.au/Infectious/alerts/Documents/EV_Surv_Report_8_ to_23June2013.pdf

16. Carver S, Bestall A, Jardine A, Ostfeld RS. Influence of hosts on the ecology of arboviral transmission: potential mechanisms influencing dengue, Murray Valley Encephalitis, and Ross River Virus in Australia. Vector Borne Zoonotic Dis. 2009;9(1):51-64.

17. Cumming G, Khatami A, McMullan BJ, Musto J, Leung K, Nguyen $\mathrm{O}$, et al. Parechovirus genotype 3 outbreak among infants, New South Wales, Australia, 2013-2014. Emerg Infect Dis. 2015;21(7):1144-52.

18. Hobday LK, Thorley BR, Alexander J, Aitken T, Massey PD, Cretikos M, et al. Potential for the Australian and New Zealand paediatric intensive care registry to enhance acute flaccid paralysis surveillance in Australia: a data-linkage study. BMC Infect Dis. 2013;13:384.

19. NSW Health. Disease notification. Sydney: NSW Government; 2015 [cited 2015 Aug 17] [about 2 screens]. Available from: www.health.nsw.gov.au/ infectious/pages/notification.aspx
20. Altmann M, Wadl M, Altmann D, Benzler J, Eckmanns T, Krause $\mathrm{G}$, et al. Timeliness of surveillance during outbreak of Shiga toxin-producing Escherichia coli infection, Germany, 2011. Emerg Infect Dis. 2011;17(10):1906-09.

21. Sullivan PS, McKenna MT, Waller LA, Williamson GD, Lee LM. Analyzing and interpreting public health surveillance data. In: Lee LM, Teutsch SM, Thacker SB, St Louis ME, editors. Principles and practice of public health surveillance, 3rd ed. New York: Oxford University Press; 2010. p. 88-145.

22. Muscatello DJ, Churches T, Kaldor J, Zheng W, Chiu C, Correll P, Jorm L. An automated, broad-based, real-time public health surveillance system using presentations to hospital emergency departments in New South Wales, Australia. BMC Public Health. 2005;5:141.

23. Yazaki Y, Mizuo H, Takahashi M, Nishizawa T, Sasaki N, Gotanda $Y$, et al. Sporadic acute or fulminant hepatitis $E$ in Hokkaido, Japan, may be food-borne, as suggested by the presence of hepatitis $E$ virus in pig liver as food. J Gen Virol. 2003;84(pt 9):2351-7.

24. Krishnamurthy RS, Louis ME. Informatics and the management of surveillance data. In: Lee LM, Teutsch SM, Thacker SB, St Louis ME, editors. Principles and practice of public health surveillance, 3rd ed. New York: Oxford University Press; 2010. p. 65-87.

25. World Health Organization. Communicable disease surveillance and response systems. Guide to monitoring and evaluating. Geneva: WHO; 2006 [cited 2014 Aug 29]. Available from: www.who.int/csr/resources/publications/ surveillance/WHO_CDS_EPR_LYO_2006_2.pdf

26. Buckeridge DL. Outbreak detection through automated surveillance: a review of the determinants of detection. J Biomed Inform. 2006;40(4):370-9.

27. Chretien JP, Tomich NE, Gaydos JC, Kelley PW. Real-time public health surveillance for emergency preparedness. Am J Public Health. 2009;99(8):1360-3.

28. Guberina H, Witzke O, Timm J, Dittmer U, Muller MA, Drosten $\mathrm{C}$,Bonin $\mathrm{F}$, et al. A patient with severe respiratory failure caused by novel human coronavirus. Infection. 2014;42(1):203-6.

29. van Vliet M, Verburg IW, van den Boogaard M, de Keizer NF, Peek N, Blijlevens NM, Pickkers P. Trends in admission prevalence, illness severity and survival of haematological patients treated in Dutch intensive care units. Intensive Care Med. 2014;40:1275-84.

30. Vincent J-L, Sakr Y, Sprung CL, Ranieri VM, Reinhardt K, Gerlach H, et al. Sepsis in European intensive care units: results of the SOAP study. Crit Care Med. 2006;34(2):344-53.

\section{Copyright: (c)}

(C) 2016 Norton et al. This article is licensed under the Creative Commons Attribution-NonCommercial-ShareAlike 4.0 International Licence, which allows others to redistribute, adapt and share this work non-commercially provided they attribute the work and any adapted version of it is distributed under the same Creative Commons licence terms. See: www.creativecommons.org/licenses/by-nc-sa/4.0/ 